\title{
Running Head: Only Game in Town Fallacy: the Only Game in Town Fallacy and Alternative Explanations for Experimental Findings
}

\author{
David Trafimow ${ }^{1}$ \\ Department of Psychology, MSC 3452 \\ New Mexico State University
}

\begin{abstract}
People commit the "only game in town fallacy" when they insist that the lack of accessible alternative explanations forces acceptance of whatever hypothesis is on the table. This fallacy is familiar to philosophers because it often comes up in the context of arguments about the existence of God. However researchers in psychology often commit the fallacy too. Is it acceptable to commit the fallacy in the context of psychology hypotheses even if it is not in the context of God? I provide several possible answers to this question, and also discuss an augmented version of the fallacy.

Keywords: only game in town fallacy; alternative explanations; accepting hypotheses
\end{abstract}

\section{INTRODUCTION}

When people argue about the existence of God, an interesting sort of fallacy sometimes arises, that is commonly termed the only game in town fallacy (OGTF). The fallacy occurs when someone insists that if there is only one explanation of something on the table, that explanation must be accepted because of the absence of competing explanations. Consider the example of Person A, who points out to Person B that there is a natural phenomenon that Person B cannot explain. Person A explains it by saying, "God did it. Because you cannot explain it, God is left as the only explanation, and so the rational thing for you to do is believe in God!" Is Person B stuck? Not so! It is perfectly reasonable for Person B to reply thusly: "I cannot explain the natural phenomenon you pointed out, but that does not mean that I have to believe your explanation; you have committed the OGTF!"

But let's change over from God to psychology findings. Suppose that Researcher A obtains findings that support Hypothesis X but researcher B does not believe it anyhow. Researcher A asks, "If you do not believe Hypothesis X, what is your alternative explanation?" Is it reasonable for Researcher B to reply, "I have no alternative explanation but I think you are wrong anyhow?" If Researcher A feels that Researcher B is being unfair by being critical in the absence of an alternative explanation, is Researcher A committing the OGTF?

This is not just a hypothetical situation. Quite some time ago, when I was in graduate school, I tested two alternative hypotheses against each other and obtained what I thought was slam-dunk support for one of them over the other. When I gave a talk in front of the faculty and graduate students, as all of us had to do on occasion, a professor (who loved to give the graduate students hell) criticized my presentation on the grounds that my experiments were artificial. I replied by asking him if he could generate an alternative explanation for my findings based on his artificiality argument. He was unable to do so (as I anticipated). He then attempted to retrieve his self-respect by claiming he did not have to generate an alternative explanation, but the other professors jumped down his throat and informed him that if he wanted to criticize in a convincing manner, he did have to generate an alternative explanation. So I, the lowly graduate student, won the day.

But was my victory a valid one? When I think about the OGTF, I have to wonder if I was wrong after all, despite my support from the faculty. Why does the OGTF seem to be such a horrible mental error in the context of God, but to be reasonable, and not even a fallacy, in the context of experimental research? Somehow it jibes with intuition that a failure to generate an alternative explanation does not require that one accept the God hypothesis, but that such a failure does require that one accept a supported hypothesis in the context of experimental research. Perhaps intuition is wrong, and that the failure of a critic to come up with an alternative explanation does not rationally require that the critic must accept the hypothesis that was supported.

\footnotetext{
${ }^{1}$ Corresponding Author: dtrafimo@nmsu.edu
} 
So where do we go from here? I do not have a clear answer but I list some possibilities below. Rather than arguing for or against any of them, I would prefer for the reader to pick his or her favorite, or even think of new one.

$>$ One possible answer is that intuition is plain wrong. The OGTF applies, regardless of whether the context is an argument about God or an argument about an experimentally supported hypothesis. Whatever the context, the critic is not required to suggest an alternative explanation to reject the experimentally supported hypothesis.

Intuition is correct when we consider a Bayesian perspective, though not from the perspective of pure logic. This is because hypotheses involving God have a much lower priori probability than scientific hypotheses. Therefore, whereas it is unreasonable to require the critic to produce an alternative explanation in the context of God, it is quite reasonable to require the critic to produce one in the context of scientific research.

$>$ A possible problem with the foregoing possibility is the premise that hypotheses involving God have a lower $a$ priori probability than research hypotheses. How would one go about establishing the truth of such a premise? So Possibility 3 would be to come to the same conclusion but for a different reason. For example, perhaps we can agree on a "rules of science" argument that might go something like the following. Anyone with a sophisticated view of science knows that it is impossible to prove anything, beyond any doubt whatsoever, based on data. So it is always possible to say, "I don't believe it." But this is too easy and gives too many advantages to the critic. In general, it is easier to tear down than to build up, and so it is inadvisable to make life even more difficult for the researcher by giving the critic the unanswerable power to reject the hypothesis without even having to provide a good reason. Thus, this is really a fair play argument. To level the playing field somewhat, the critic should at least be required to provide a reason for rejecting the researcher's experimentally supported hypothesis, other than because the critic doesn't like it. That reason likely will take the form of an alternative explanation for the data (though it could take other forms, such as pointing out an internal contradiction in the researcher's logic). In sum, then, because of the OGTF, the critic who cannot come up with a decent reason for rejection might not be required by strict logic to give in to the researcher's hypothesis, but the critic nevertheless would be required to acknowledge defeat by the rules of fair play in science.

$>$ Note that this fair play argument would not necessarily apply to God. After all, it does not take much creativity to shout, "God did it!" And so it is possible to argue that what is fair differs in the context of God versus the context of scientific hypotheses.

$>$ Yet another possibility is to be nuanced about the OGTF and argue that acceptance or rejection of the hypothesis depends on the argument that the researcher is trying to make. Consider the difference between two ways of supporting Hypothesis X. One way is to simply show that the data are consistent with what Hypothesis X would predict. A second way is to show that whereas Hypothesis $\mathrm{X}$ is consistent with the data, Hypothesis $\mathrm{Y}$ is contradicted by the data. In the first example, the argument is simply that we should accept hypothesis $\mathrm{X}$ in some absolute sense, because there is a lack of a competing hypothesis to generate a competitive context. In this case, perhaps, committing the OGTF really is bad practice whereas, in contrast, there is a different conclusion that one can come to in the second example. Specifically, in the second example, it seems reasonable to come to the modest conclusion, so long as it is a tentative one pending future research, that Hypothesis $\mathrm{X}$ is superior to Hypothesis Y. In this case, if the critic cannot generate an alternative explanation, it seems inescapable that one has to give the advantage to Hypothesis X over Hypothesis Y. Of course, if the critic can generate an alternative explanation it might still be possible to reasonably uphold Hypothesis $\mathrm{Y}$ as a plausible competitor.

Stated more generally, then, Possibility 4 states that the failure to generate an alternative hypothesis does not force a critic to accede when only one hypothesis is tested; this would be a commission of the OGTF. But suppose that one hypothesis wins out over the other in an experimental test, and no alternative explanations are generated. In that case, although critic does not have to accept the winning hypothesis in an absolute sense because that would be committing the OGTF, the fair critic must at least admit that the winning hypothesis is superior to the losing one pending the acquisition of more data.

\section{The AUgMented OGTF}

Perhaps it is worthwhile to consider an augmented version of the OGTF that can be illustrated by an experience that I have had on multiple occasions. In my most recent of OGTF encounters, I obtained some data that disconfirmed, in a very direct way, a widely held hypothesis. Reviewers who were aficionados of the hypothesis claimed that because I did not offer alternative explanations of their findings, I did not actually disconfirm the hypothesis, despite the fact that the hypothesis made predictions that were demonstrated to be wrong in my experiments. In essence, the argument was that it is insufficient to show that predictions of a hypothesis are wrong. Rather one has to produce an 


\section{American Research Journal of Humanities and Social Sciences, Volume 1, Issue1, Feb-2015 ISSN 2378-7031}

explanation for the findings that are consistent with it, in addition to showing that the hypothesis makes wrong predictions, before it is disconfirmed. Because I only showed that the hypothesis made wrong predictions, but I failed to propose alternative explanations for the findings in the literature that supported the hypothesis, committing the OGTF implies that I failed to actually disconfirm the hypothesis!

This augmented OGTF is perhaps even worse than the base version. According to the base version of the OGTF, the lack of an alternative explanation necessitates acceptance of the hypothesis, which is bad enough, but in this augmented version, a lack of alternative explanations forces acceptance of the hypothesis even in the face of contradictory data!

\section{Conclusion, Policy ImPlications And Limitations}

A common way to criticize research in psychology is via the proposal of alternative explanations for the findings. I have no profound disagreement against this, except that reviewers sometimes are not sufficiently careful to make sure these alternative explanations are not contradicted by data other than the data they are considering at the time. My goal was to examine the inverse argument, that a lack of alternative explanations buffers a hypothesis against non-acceptance. On the contrary, I stated that making this leap does not conform to strict logic, and is an example of the OGTF. Is it reasonable to commit the OGTF in the context of science, whereas it might be less permissible in the context of God? I suggested multiple ways to address this question. Finally, I suggested the augmented OGTF as a more pernicious form of the fallacy.

\section{REFERENCES}

Sober, E. (2001). Core questions in philosophy. Upper Saddle River, N. J.: Prentice Hall. 